were modestly less painful than larger tubes (adjusted mean difference, $-6.0 \mathrm{~mm}(95 \% \mathrm{CI},-11.7$ to $-0.2 ; \mathrm{p}=0.04))$ and were associated with a higher pleurodesis failure rate which failed to meet non-inferiority criteria (pleurodesis failure 15/50 (30\%) and $12 / 50(24 \%)$ respectively, difference $6 \%(90 \% \mathrm{CI},-9 \%$ to $20 \%)$ ). Adverse events did not differ between analgesic groups, but complications during insertion occurred more commonly with smaller drains (adjusted odds ratio, 1.91; 95\% CI 0.71 to $5.13, \mathrm{p}=0.20$ ).

Conclusion NSAID and opiate analgesia were not significantly different in treatment of post-pleurodesis pain and neither was associated with impaired efficacy of pleurodesis. Smaller chest tubes were associated with less pain, but may be associated with reduced pleurodesis success compared with larger tubes. These results challenge current guidelines for pleurodesis of MPE, which advocate avoidance of NSAID and use of small chest tubes.

\section{S21 EARLY CONTRAST ENHANCEMENT: A PERFUSION- BASED MAGNETIC RESONANCE IMAGING BIOMARKER OF PLEURAL MALIGNANCY}

${ }^{1} \mathrm{~S}$ Tsim, ${ }^{1} \mathrm{CA}$ Humphreys, ${ }^{1} \mathrm{DB}$ Stobo, ${ }^{1} \mathrm{GW}$ Cowell, ${ }^{2} \mathrm{R}$ Woodward, ${ }^{2} \mathrm{JE}$ Foster, ${ }^{1} \mathrm{C}$ Dick, ${ }^{1} \mathrm{KG}$ Blyth. 'Queen Elizabeth University Hospital, Glasgow, UK; ${ }^{2}$ Glasgow Clinical Research Imaging Facility, Glasgow, UK

\subsection{6/thoraxjnl-2015-207770.27}

Introduction and objectives Radiological detection of pleural malignancy (PM) remains challenging. In early-stage Malignant Pleural Mesothelioma (MPM) a pleural effusion may be the only significant abnormality, indistinguishable from benign asbestos-related pleural effusion (BAPE). PM is associated with neovascularisation. We report the diagnostic performance of a novel perfusion-based Magnetic Resonance Imaging (MRI) biomarker of PM - Early Contrast Enhancement (ECE).

Methods 24 patients with suspected PM were recruited prospectively. All underwent contrast-enhanced Computed Tomography (CT) scanning, 3T Pleural MRI and Thoracoscopy.

18/24 had complete MRI examinations: T1-weighted 3Dspoiled-gradient-echo sequences acquired at baseline, $40 \mathrm{~s}, 80 \mathrm{~s}$ and 4.5, 9 and 13.5 min after intravenous Gadobutrol contrast. Mean signal intensity (SI) of representative parietal pleura was derived from 15 regions of interest placed by two respiratory physicians. ECE was defined objectively by an early peak in mean SI $(\leq 4.5 \mathrm{~min})$ on the resulting SI/time curve (Figure 1). Morphology suggestive of PM on CT and MRI was recorded by two thoracic radiologists. Diagnostic performance and interobserver agreement for ECE, MRI and CT morphology were compared. All analyses were blinded.

Pleural SI data were correlated against Microvessel Density (MVD) measured in paraffin-embedded pleural biopsies stained with CD34 and Factor VIII immunostains.

Results Mean patient age was 73 (SD 8) years. 18/24 were asbestos-exposed and 12/18 had pleural thickening $\leq 5 \mathrm{~mm}$. ECE was present in 10/11 patients with PM (MPM ( $\mathrm{n}=10)$; lung cancer $(\mathrm{n}=1))$. The false negative case had MPM. ECE was absent in 6/7 patients with benign pleural disease (BAPE $(n=4)$, fibrothorax $(\mathrm{n}=2)$, TB $(\mathrm{n}=1))$. The false positive case had TB.

Overall diagnostic accuracy of ECE, MRI and CT morphology: sensitivity 91\%, 91\%, 90\%; specificity 86\%, 71\%, 50\%; negative predictive value $86 \%, 83 \%, 80 \%$; positive predictive value 91\%, 83\%, 69\% respectively. Inter-observer agreement was 0.766 for ECE, 0.727 for MRI and 0.753 for CT.

Figure 1 shows the relationship between MVD and Pleural SI.

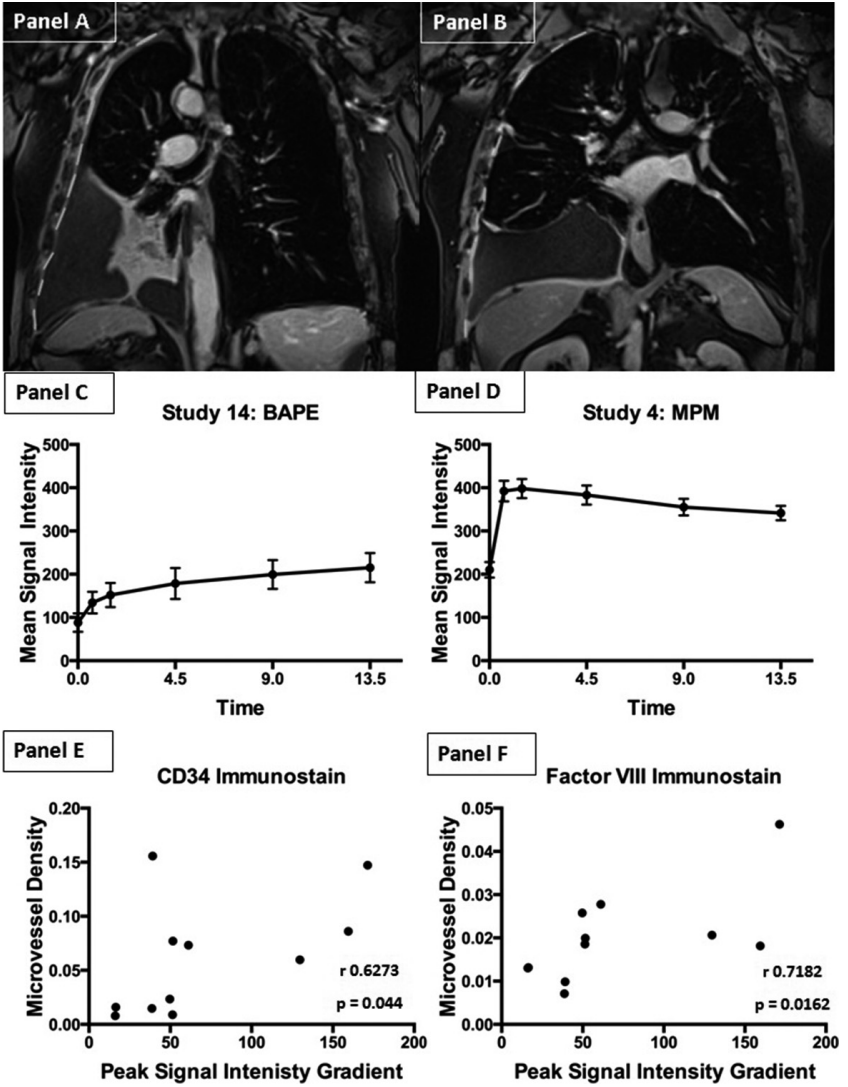

Abstract S21 Figure 1 Example of 3D T1-weighted Pleural MR images acquired 4.5 minutes post-Gadobutrol in 2 different patients one with BAPE (Panel A) and one with early-satge MPM (Panel B). Panels $C$ and $D$ show the accompanying partietal pleura SI/time curves for each patient (Panel C: patient with BAPE - ECE absent, panel D: patient with MPM - ECE present). Panels E and F demonstrate the relationship between peak signal intensity gradient in patients with pleural malignancy and Microvessel Density measured in diseased tissue with CD34 immunostain (Panel E) and Factor VIII immunostain (Panel F)

Conclusions ECE appears an accurate and reproducible, perfusion-based, objective biomarker of PM, out-performing subjectively-defined CT and MR morphology. ECE assessment can be performed in patients with minimal pleural thickening, suggesting potential utility as a biomarker of early-stage MPM or low-volume metastatic PM.

\section{S22 VATS FOR PRIMARY SPONTANEOUS PNEUMOTHORAX - A COHORT STUDY OF 1415 PATIENTS}

${ }^{1} \mathrm{G}$ Cardillo, ${ }^{2} \mathrm{OJ}$ Bintcliffe, ${ }^{1} \mathrm{~F}$ Carleo, ${ }^{2} \mathrm{NA}$ Maskell. ${ }^{1}$ Carlo Forlanini Hospital, Rome, Italy; ${ }^{2}$ University of Bristol, Bristol, UK

\subsection{6/thoraxjnl-2015-207770.28}

Background Video-assisted thoracic surgery (VATS) is an increasingly common treatment for recurrent or persistent primary spontaneous pneumothorax (PSP). Surgery usually involves a diffuse treatment of the pleura and possible targeted surgical techniques on areas of bullous disease. There is no robust evidence to guide the use of specific surgical techniques. The purpose of this large observational cohort study was to examine the recurrence rates associated with VATS and identify predictors of outcome. 
Methods Patients undergoing VATS for PSP at Carlo Forlanini hospital in Rome between January 2000 and December 2012 were prospectively collected. All patients underwent talc poudrage. Targeted surgical techniques were selected based on the presence of air leak and Vanderschueren stage. Patients had regular clinical and radiological follow-up for a minimum of 2 years. Surgical details, demographics and smoking histories were collected at baseline and data on duration of hospital stay, complications and recurrence rates were collated.

Results 1415 patients underwent VATS for PSP during the trial period. The majority of patients were male (76.2\%). Median age was 25.3 years (IQR 21.0-29.4). The majority of patients underwent surgery due to recurrent pneumothorax (92.2\%). Median length of stay was 5 days (IQR 5-6). 47 patients had incomplete follow up in December 2014 and so complete recurrence data is available for 1368 patients.

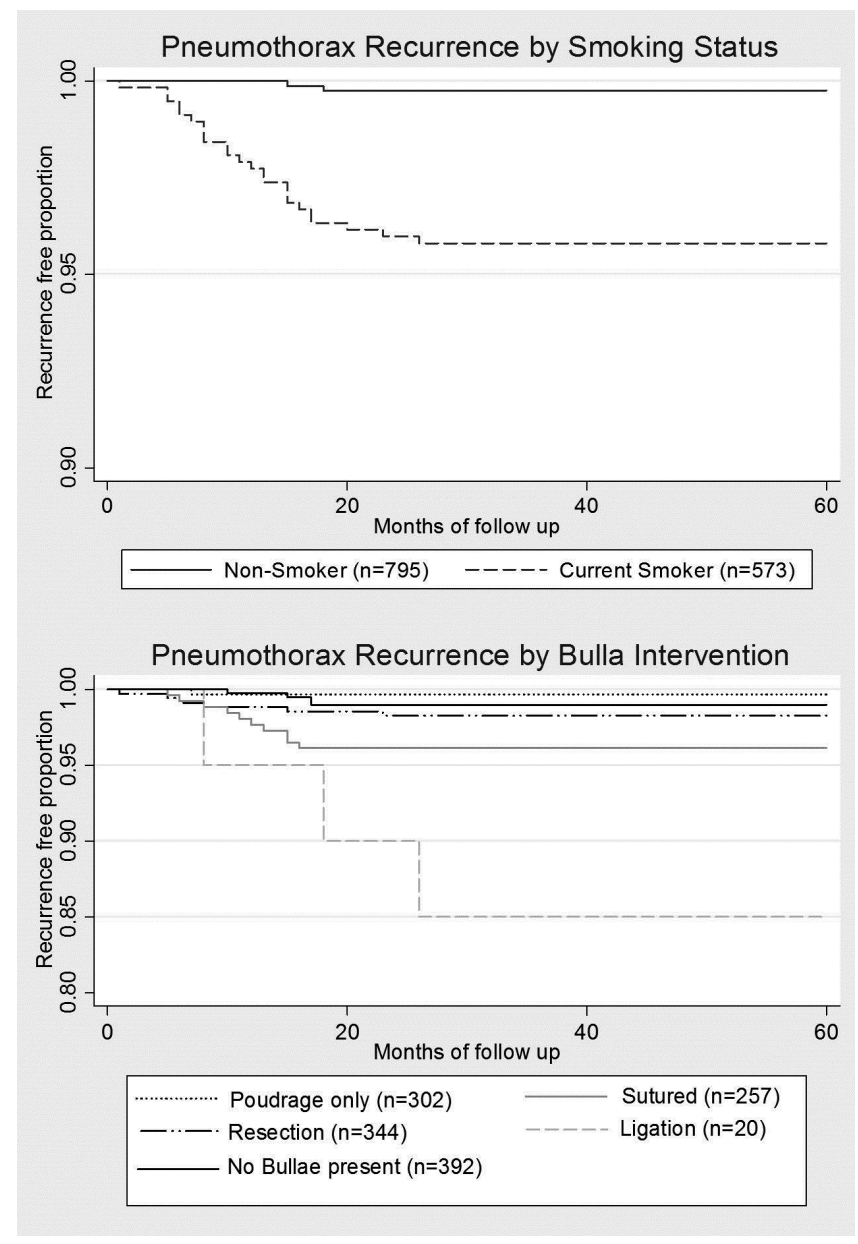

\section{Abstract S22 Figure 1}

VATS had a low complication rate of $2 \%$, the majority of which was prolonged air leak (1.7\%). Recurrent pneumothorax occurred in 26 patients (1.9\%) over a median follow up of 8.5 years. Recurrence rates were significantly higher in current smokers at the time of surgery $(24 / 573-4.2 \%)$ than in nonsmokers $(2 / 796-0.25 \%) \mathrm{p}<0.001$. Bullae suturing (3.9\%) and ligation $(15 \%)$ were associated with statistically significant higher rates of recurrence compared with poudrage alone when controlled for smoking status and Vanderschueren stage.

Conclusions The marked difference in recurrence rates between smokers and non-smokers suggests that this factor is of key importance in predicting recurrence risk after VATS. This study demonstrates a low incidence of recurrence for patients undergoing VATS for PSP. Bullae ligation and bullae suturing appear to be associated with a higher risk of recurrence.

\section{S23 AMBULATORY PERCUTANEOUS LUNG BIOPSY WITH EARLY DISCHARGE AND HEIMLICH VALVE MANAGEMENT OF IATROGENIC PNEUMOTHORAX - A NEW MODEL FOR THE UK}

RR Abdullah, AN Tavare, DD Creer, S Khan, R Vancheeswaran, SS Hare. Barnet General Hospital, Royal Free London NHS Foundation Trust, London, UK

\subsection{6/thoraxjnl-2015-207770.29}

Aim To determine if an early discharge radiology-led percutaneous lung biopsy (PLB) service, incorporating ambulatory outpatient small calibre Heimlich valve chest drain (HVCD) to treat pneumothorax, is potentially safe and advantageous to the NHS. Methods A prospective study of 489 consecutive outpatient image-guided PLBs, performed between March 2011-March 2015, was conducted. Patients were discharged at $30 \mathrm{~min}$ if no pneumothorax was present; repeat 60-minute CXR was performed if a small asymptomatic pneumothorax was noted. If stable, patients were discharged. In enlarging or symptomatic pneumothorax, patients were discharged with HVCD in situ and followed up for drain removal. Data on complications was concurrently collected, including pneumothorax rates, numbers of patients requiring HVCD and failed early discharge. A retrospective blinded pulmonary function test (PFT) analysis was also performed at the end of the study period.

Results 489 PLBs were performed with diagnostic accuracy of 97.8\%. $402(82.2 \%)$ patients were discharged at $30 \mathrm{~min}$, all without further incident. 87 patients developed pneumothorax (17.8\%). 35 patients with a small stable, asymptomatic pneumothorax were discharged at $60 \mathrm{~min}$ without complication. 52 patients required HVCD, with $5 / 52$ proceeding to PLB with drain in-situ: $38 / 52(73.1 \%)$ had drain removal at $24 \mathrm{~h}$ and $14 /$ $52(26.9 \%)$ at $48 \mathrm{~h}$, with none requiring HVCD greater than 48 h. 4/489 patients were admitted, for social issues.

A blinded retrospective review of PFT data, available in 212/ 489 patients, revealed 28 with FEV1 < 11. 22/28 (78.6\%) were discharged at $30 \mathrm{~min}$ without incident; 6/28 patients (21.4\%) developed post -PLB pneumothorax with three (10.7\%) requiring outpatient HVCD, for $24 \mathrm{~h}$ duration.

Conclusion This prospective study of 489 consecutive outpatient PLBs, novel in the NHS setting, provides evidence for a paradigm shift in current UK lung biopsy practice: (i) early discharge PLB, facilitated by use of ambulatory HVCD, is safe and expeditious, thereby enabling more prompt lung cancer diagnosis; and (ii) use of outpatient HVCD is clinically and economically beneficial, saving precious hospital beds whilst also facilitating lung biopsy in severely emphysematous patients with negligible morbidity.

\section{S24 LUNG PARENCHYMAL ASSESSMENT IN PRIMARY AND SECONDARY PNEUMOTHORAX - A CASE-CONTROL STUDY}

${ }^{1}$ OJ Bintcliffe, ${ }^{2}$ AJ Edey, ${ }^{3}$ IS Negus, ${ }^{1}$ NA Maskell. 'University of Bristol, Bristol, UK; ${ }^{2}$ North Bristol NHS Trust, Bristol, UK; ${ }^{3}$ University Hospitals Bristol NHS Foundation Trust, Bristol, UK

10.1136/thoraxjn-2015-207770.30 